\title{
NORMAL MATRIX COMPRESSIONS
}

\author{
John Holbrook, Nishan Mudalige and Rajesh Pereira
}

Abstract. There has been longstanding interest in the problem of characterizing normal compressions of normal matrices. Indeed, the solution to the Hermitian case goes back to the Cauchy interlacing theorem, and its converse (due to Fan and Pall). More recently, the theory of higherrank numerical ranges has included the solution in the case of scalar compressions. Here we take steps towards a similar treatment of the general case. We develop some natural necessary conditions on the eigenvalues as well as some convenient sufficient conditions, showing by a study of the $2 \times 2$ compressions of $4 \times 4$ normals that the necessary conditions are not sufficient. We also give a new proof of the Choi-Kribs-Życzkowski conjecture for $2 \times 2$ compressions by means of a powerful extension of that result. The CKŻ conjecture (more recently a theorem) may be stated as follows: given an $N \times N$ normal matrix $M$ with eigenvalues $\lambda_{1}, \ldots, \lambda_{N}$, the set of $a \in \mathbb{C}$ for which the scalar matrix $a I_{k}$ is a compression of $M$ is precisely

$$
\Omega_{k}(M)=\bigcap_{\#(J)=N-k+1} \operatorname{conv}\left\{\lambda_{j}: j \in J\right\} .
$$

Thus, for $k=2$ we see that $a \in \Omega_{2}(M)$ implies that $\operatorname{diag}(a, a)$ is a compression of $M$ (the reverse implication is relatively straightforward). We show that, in fact, for any pair $a, b \in$ $\Omega_{2}(M), \operatorname{diag}(a, b)$ is a compression of $M$. Our proof is independent of the earlier results and depends on a novel approach. We also study the continuity of the map $a \rightarrow B(a)$, where $B(a)$ denotes the set of all $b \in \mathbb{C}$ such that $\operatorname{diag}(a, b)$ is a compression of $M$.

Mathematics subject classification (2010): 47A12, 15A60, 15A90, 81P68.

Keywords and phrases: Matrix compression, higher-rank numerical ranges, interlacing theorems.

\section{REFERENCES}

[1] W. ARveson, Unitary invariants for compact operators, Bull, Amer. Math. Soc. 76, 88-91, 1970.

[2] K. Audenaert and F. Kittaneh, Problems and conjectures in matrix and operator inequalities, arXiv:1201.5232v2 [math.FA], 2012.

[3] A. L. CAUChy, Sur l'équation à l'aide de laquelle on détermine les inégalités séculaires des mouvements des planètes, Oeuvres complètes, Second Ser., IX, 174-195.

[4] M.-D. ChOI, D. W. KRIBS, AND K. ŻYCZKOWS KI, Higher-rank numerical ranges and compression problems, Linear Algebra Appl. 418, 828-839, 2006.

[5] M.-D. ChOI, D. W. KRIBS, AND K. ŻYCZKOws KI, Quantum error correcting codes from the compression formalism, Rep. Math. Phys. 58, 77-91, 2006.

[6] M.-D. Choi, J. A. Holbrook, D. W. Kribs, And K. ŻYczkowski, Higher-rank numerical ranges of unitary and normal matrices, Operators and Matrices 1, 409-426, 2007.

[7] M.-D. Choi, M. Giesinger, J. A. Holbrook, And D. W. Kribs, Geometry of higher-rank numerical ranges, Linear and Multilinear Algebra 56, 53-64, 2008.

[8] W. E. Chum, The unitary equivalence of compact operators, Glasgow Math. J. 26, 145-149, 1985.

[9] C. F. Dunkl, P. Gawron, J. Holbrook, Z. Puchala, and K. ŻYCZKowski, Numerical shadows: measures and densities on the numerical range, Linear Algebra Appl. 434, 2042-2080, 2011.

[10] K. FAN AND G. PALL, Imbedding conditions for Hermitian and normal matrices, Canadian J. Math. 9, 298-304, 1957. 
[11] D. R. FARENICK, Normal compressions and restrictions to 2-dimensional subspaces, and the Berberian spectral extension, Special Issue: The numerical range and numerical radius, Linear and Multilinear Algebra 37, \#1-3, 131-138, 1994.

[12] P. Gawron, Z. Puchala, J. Miszczak, L. Skowronek, and K. Życzkowski, Restricted numerical range: a versatile tool in the theory of quantum information, J. Math. Physics 51, 2010.

[13] J. A. Holbrook, N. Mudalige, And R. Pereira, Normal matrix compressions, arXiv:1203.3908v2 [math.FA], 2012.

[14] D. W. Kribs, A. Pasieka, M. Laforest, C. Ryan, And M. P. Da Silva, Research problems on numerical ranges in quantum computing, Linear and Multilinear Algebra 57, 491-502, 2009.

[15] C.-K. Li AND Y.-T. Poon, Generalized numerical ranges and quantum error correction, J. Operator Theory 66, 335-351, 2011.

[16] C.-K. LI, Y.-T. Poon, AND N.-S. SZE, Higher rank numerical ranges and low rank perturbations of quantum channels, J. Math. Analysis Appl. 348, 843-855, 2008.

[17] C.-K. LI, Y.-T. Poon, AND N.-S. SZE, Condition for the higher rank numerical range to be nonempty, Linear and Multilinear Algebra 57, 365-368, 2009.

[18] C.-K. Li AND N.-S. SzE, Canonical forms, higher-rank numerical ranges, totally isotropic subspaces, and matrix equations, Proc. Amer. Math. Soc. 136, 3013-3023, 2008.

[19] C.-K. Li AND N.-K. Tsing, On the kth matrix numerical range, Linear and Multilinear Algebra 28, \#4, 229-239, 1991.

[20] K. MAJgier, H. MAASSEn, AND K. ŻYCZKows Ki, Protected subspaces in quantum information, Quantum Information Processing 9, 343-367, 2010.

[21] N. Mudalige, Higher Rank Numerical Ranges of Normal Operators, MSc thesis, U of Guelph, 2010.

[22] F. M. Pollack, Properties of the matrix range of an operator, Indiana Univ. Math. J. 22, 419-427, 1972/73.

[23] J. F. QueIRó AND A. L. DuARTE, Imbedding conditions for normal matrices, Linear Algebra Appl. 430, 1806-1811, 2009.

[24] R. C. ThOmpson, Research problem: The matrix numerical range, Linear and Multilinear Algebra 21, 321-323, 1987.

[25] J. P. Williams, On compressions of matrices, J. London Math. Soc. (2) 3, 526-530, 1971.

[26] H. Woerdeman, The higher-rank numerical range is convex, Linear and Multilinear Algebra 56, 65-67, 2008. 